\title{
Structural properties of liposomes from digital holographic microscopy
}

\author{
Isabelle L. Di Maio ${ }^{\mathrm{a}}$, Daniel Carl ${ }^{\mathrm{b}}$, Patrik Langehanenberg ${ }^{\mathrm{b}}$, Stella M. Valenzuela ${ }^{\mathrm{a}}$, \\ Andrew R. Battle ${ }^{a}$, Sabah Al Khazaaly ${ }^{a}$, Murray Killingsworth ${ }^{c}$, Björn Kemper ${ }^{b}$, \\ Gert von Bally ${ }^{\mathrm{b}}$, Donald K. Martin ${ }^{*}$ \\ ${ }^{a}$ Department of Health Science, University of Technology Sydney, P.O. Box 123, Broadway N.S.W. \\ 2007, Australia \\ ${ }^{\mathrm{b}}$ Laboratory of Biophysics, Medical Centre, University of Münster, Robert-Koch-Str. 45, 48129 \\ Münster, Germany \\ ${ }^{\mathrm{c}}$ Department of Anatomical Pathology, South Western Area Pathology Service, Liverpool Hospital, \\ Liverpool, NSW 2170, Australia.
}

\begin{abstract}
We have constructed liposomes from L-alpha-Phosphatidylcholine (PC) lipids, which are biomimetic lipids similar to those present in the membranes of mammalian cells. We propose an advance in the use of liposomes, such as for drug delivery, to incorporate into the liposomal membranes transport proteins that have been extracted from the lipid membranes of mammalian cells. In this paper, we describe the usage of a novel optical microscope to characterize the nanomechanical properties of these liposomes. We have applied the technique of digital holographic microscopy ${ }^{1}$, using an instrument recently developed at the University of Münster, Germany. This system enabled us to measure quantitatively the structural changes in liposomes. We have investigated the deformations of these biomimetic lipids comprising these liposomes by applying osmotic stresses, in order to gain insight into the membrane environment prior to incorporation of cloned membrane transport proteins. This control of the nanomechanical properties is important in the stresses transmitted to mechanosensitive ion channels that we have incorporated into the liposomal membranes. These liposomes provide transporting vesicles that respond to mechanical stresses, such as those that occur during implantation.
\end{abstract}

Keywords: Giant liposomes, unilamellar, multilamellar, digital holographic microscopy

\section{INTRODUCTION}

The construction of liposomes which are vesicular structures composed of phospholipid bilayers have attracted considerable interest since their discovery in the $60^{\prime} \mathrm{s}^{2,3}$. They provide a model for biological membranes due to their organization and the versatility of their physicochemical properties. In addition, liposomes are useful nano and microscale vesicles for biomedical applications ${ }^{4-6}$, for example drug delivery and targeting ${ }^{7,8}$. Liposomes are potential carriers for a variety of drugs that include therapeutic proteins and diagnostic agents. Indeed, membrane transport proteins can be incorporated in the liposomal membrane and act as multidrug transporters ${ }^{9}$. But prior to incorporation of these transport proteins, it is necessary to gain insight into the membrane environment of these vesicles.

Amongst the numerous procedures that have been developed to prepare liposomes from biomimetic lipids ${ }^{10,11}$, the formation of giant unilamellar vesicles, either by hydration or electroformation ${ }^{12}$, has proved to be valuable. The size of the liposomes, similar to those of living cells ${ }^{13}$, enables their visualization by optical microscopy and the micromanipulation of individual vesicles ${ }^{14}$. These large vesicles have been widely used to study lipid mobility ${ }^{15}$, membrane dynamics ${ }^{16}$, lipid domain formation ${ }^{17}$, lipid-DNA interactions ${ }^{18}$, vesicle shape change ${ }^{19}$, membrane curvature and elasticity ${ }^{20}$. Undeniably, biological membranes are highly dynamic systems and can deform themselves in the case of external stresses. Numerous articles have been dedicated to these geometric transformations which imply physical properties such as lipid fluidity, stretching and bending elasticity. Méléard et $a .^{21}$ gave bending elasticities as a function

\footnotetext{
*donm@uts.edu.au; phone +61 29514 2232; fax +61 295142228
} 
of sterol content and temperature. Viallat et al. ${ }^{22}$ studied the shape instability in giant liposomes induced by osmotic shrinkage and compared the results with the shapes predicted from the area difference elasticity model ${ }^{23}$. Typically, the mechanical properties of giant liposomes have been analysed by optical microscopy. Microscopic measurement has been combined with fluorescence measurement to correlate shape changes with lateral phase separation within the membrane ${ }^{24}$. Other studies demonstrate the usage of phase contrast microscopy ${ }^{25}$ or pulse-light microscopy ${ }^{26}$.

As a novel optical method, holographic interferometric microscopy has been recently developed at the University of Münster, Germany ${ }^{1}$. This instrument allows a fast, non destructive and quantitative high resolution full field detection of optical path length changes. With a measurement accuracy in the sub micrometer range, interferometry has already shown its application in medicine and biology $y^{27}$. In combination with microscopic resolution, the digital holographic setup offers new possibilities for detecting refractive index changes and variations in the shape of unlabelled cellular samples. Digital holographic microscopy could be applied to a marker free monitoring of life processes and cell manipulation ${ }^{28}$ as well as automated cell differentiation.

In digital holography, the phase information that is reconstructed simultaneously to the absolute amplitude can be applied for quantitative phase contrast microscopy ${ }^{1}$. It is the reason why we have investigated osmotic stresses affecting liposomes, such as swelling or shrinking.

In the present work, we report initially the construction of giant liposomes as either multilamellar or unilamellar vesicles. Giant unilamellar liposomes were prepared using sucrose solutions of various concentrations. Sucrose is normally used for cryoprotection of cells and liposomal solutions with different forms of saccharides naturally found in association with cell membranes. It is known from calorimetry and x-ray diffraction that sucrose does not significantly alter lipid bilayer packing or phase transition temperature, except at extreme dehydration ${ }^{29,}{ }^{30}$. Subsequently, we demonstrate the shrinking process of a giant unilamellar liposome by the addition of a $2 \mathrm{M} \mathrm{NaCl}$ solution using digital holographic microscopy. In order to monitor the deformation of the giant vesicle, a measurement cell with a large solution chamber was developed. Tubing was added to the cell to allow the solution of salt to enter the chamber at the desired flow rate.

\section{MATERIALS AND METHODS}

\subsection{Chemicals}

L-alpha-phosphatidylcholine (PC) from soybean, cholesterol, chloroform, sucrose, glucose, sodium chloride (NaCl), potassium chloride $(\mathrm{KCl})$ and 4-(2-Hydroxyethyl)piperazine-1-ethanesulfonic acid (HEPES) were purchased from Sigma Chemical Co. (St Louis, MO, USA). 5-dodecanoylaminofluorescein from Invitrogen (Carlsbad, USA) was first dissolved in dimethyl sulphoxide (DMSO) at a concentration of $10 \mathrm{mg} \cdot \mathrm{mL}^{-1}$ and later diluted to $1 \mathrm{mg} \cdot \mathrm{mL}^{-1}$ in ethanol. Water of high resistivity (18.2 M' $\Omega$ ) was freshly prepared using a Milli-Q system from Millipore (Belford, MA, USA).

\subsection{Preparation of giant liposomes}

Giant multilamellar liposomes were prepared by first diluting PC lipid $\left(100 \mathrm{mg} \cdot \mathrm{mL}^{-1}\right)$ and cholesterol (either 10 or 40 $\mathrm{mg} \cdot \mathrm{mL}^{-1}$ ) in chloroform to produce a mixture of $400 \mu \mathrm{L}$ in a glass test tube. The mixture was dried at room temperature by a stream of nitrogen gas to form a thin lipid film upon the surface of the tube. The tube was subsequently placed in vacuo for $>4 \mathrm{~h}$ to remove the last trace of organic solvent. The lipids were then resuspended in buffer $(200 \mathrm{mM} \mathrm{KCl} / 5$ $\mathrm{mM}$ HEPES, $\mathrm{pH}$ 7.2) and bath-sonicated for $15 \mathrm{~min}$. The liposomes were collected by ultracentrifugation at $40,000 \times \mathrm{g}$ for $40 \mathrm{~min}$ and resuspended in $300 \mu \mathrm{L}$ buffer. $40 \mu \mathrm{L}$ of this liposomes solution was spotted onto a glass plate and left to dry under vacuum for 4-6 h. Giant multilamellar liposomes were then formed by overnight rehydration in buffer.

Giant unilamellar liposomes were prepared according to the standard hydration procedure $25,31,32$ with some modifications. In brief, a completely dried lipid film containing $100 \mu \mathrm{L}$ of $10 \mathrm{mg} \cdot \mathrm{mL}^{-1}$ PC with $10 \%(\mathrm{w} / \mathrm{w}$ ) or $40 \%$ $(\mathrm{w} / \mathrm{w})$ cholesterol in chloroform was prepared in a glass test tube as described earlier. Rehydration of the lipid was later made at $45^{\circ} \mathrm{C}$ by the addition of a small amount of pure water $(5 \mu \mathrm{L})$ to the tube for a few minutes (prehydration) followed by the addition of $5 \mathrm{~mL}$ of an aqueous solution of $0.1 \mathrm{M}$ or $2 \mathrm{M}$ sucrose. The tube was incubated at $45^{\circ} \mathrm{C}$ for 2- 
$3 \mathrm{~h}$. After gentle rocking overnight at room temperature, the lipid film dispersed uniformly in the solution and a white cloud was floating in the middle of the solution, which contained giant liposomes. The obtained liposomes were stable up to four days at $4^{\circ} \mathrm{C}$.

\subsection{Observation of liposomes}

Giant unilamellar liposomes were observed through a $60 \times$ water immersion objective on a confocal laser scanning microscope (FV300, Olympus, Tokyo, Japan ) in transmitted light and fluorescent modes. To increase the contrast of the membrane, $5 \mu \mathrm{L}$ of giant unilamellar liposomes were diluted into $300 \mu \mathrm{L}$ of $0.1 \mathrm{M}$ or $2 \mathrm{M}$ aqueous glucose solution. The replacement of the external solution with an equimolar glucose solution allowed the sedimentation of the liposomes (the density of sucrose and glucose at $20^{\circ} \mathrm{C}$ are 1.35 and $1.07 \mathrm{~g} \cdot \mathrm{mL}^{-1}$, respectively). Fluorescent images were obtained using argon laser (488 nm excitation, with 510 high band pass emission filter). To stain the lipid membrane, $5 \mu \mathrm{L} \mathrm{of} 1 \mathrm{mg} \cdot \mathrm{mL}^{-1}$ fluorescein dye solution were added to the diluted solution of giant unilamellar liposomes.

To further assess the lamellarity of the membrane, Transmission Electron Microscopy (TEM) images of unilamellar and multilamellar liposomes were obtained at the Department of Anatomical Pathology, Liverpool Hospital (NSW, Australia) after dilution by a factor of 2 in fixative agent, generously donated by that Department.

\subsection{Digital holographic microscopy}

Fig. 1A shows the schematic of the digital holographic microscopy setup. The emitted light of a frequency doubled Nd:YAG laser $(\lambda=532 \mathrm{~nm})$ is divided into object wave and reference wave. The coherent illumination of the probe is realized in inverse transmission light arrangement. For that purpose, the object wave is integrated into the optical path of an inverse microscope (Zeiss Axiovert) by a polarizing beam splitter cube. The condenser is adjusted as for conventional white light illumination. A 40× microscope objective (Zeiss Acroplan 40x, NA 0.6 LD) is used for investigation of the giant liposomes in the measurement cell, to magnify the object wave. To arrange holographic "off-axis" geometry, a slightly tilted reference wave is superimposed to the object wave by a second beam splitter cube. Digital holograms are captured by an 8 bit CCD array with IEEE 1394 interface.

Numerical reconstruction of the object wave is performed in two steps. In a first step, the complex object wave (amplitude and phase) is reconstructed by solving the interferogram equation within the hologram plane (CCD array). If necessary, due to defocused object structures, the complex wave is propagated in a second step. In this step, numerical propagation is performed by evaluation of the Fresnel-Kirchhoff diffraction integral, e.g. with the discrete Fresnel transform (DFT). The combination of image plane holography with wave front propagation enables the reconstruction of multiple focus planes without the disturbing term: twin image and zero order. As a result, the full pixel resolution of the CCD sensor is available for the reconstructed object wave ${ }^{1}$. From the reconstructed complex object wave, in addition to the absolute amplitude that represents the image of the sample, the phase information of the object wave is obtained modulo $2 \pi$ simultaneously. After removal of this ambiguity by an unwrapping process the obtained data can be applied for quantitative phase contrast microscopy.

Fig. 1B represents the object cell placed on the stage of the microscope. The measurement cell used to shrink liposomes was composed of two plexiglass holders where round coverslips enclosed the chamber at the top (Ø22 $\mathrm{mm})$ and at the bottom (Ø12 mm). The giant liposomes were left to settle at the bottom of the chamber on the small round coverslip for 20 min before any measurement. The tubing allowed the solution of salt to enter the chamber. An o-ring, placed between the upper and lower part of the device, prevented any linkage from the chamber.

For the shrinking process, the giant liposomes containing $2 \mathrm{M}$ sucrose solution were diluted in $1 \mathrm{M} \mathrm{NaCl}$ solution before sedimentation. The deformation of the liposomes was later observed by adding a $2 \mathrm{M} \mathrm{NaCl}$ solution to the chamber at a flow rate of $1 \mathrm{~mL} \cdot \mathrm{min}^{-1}$. 
A

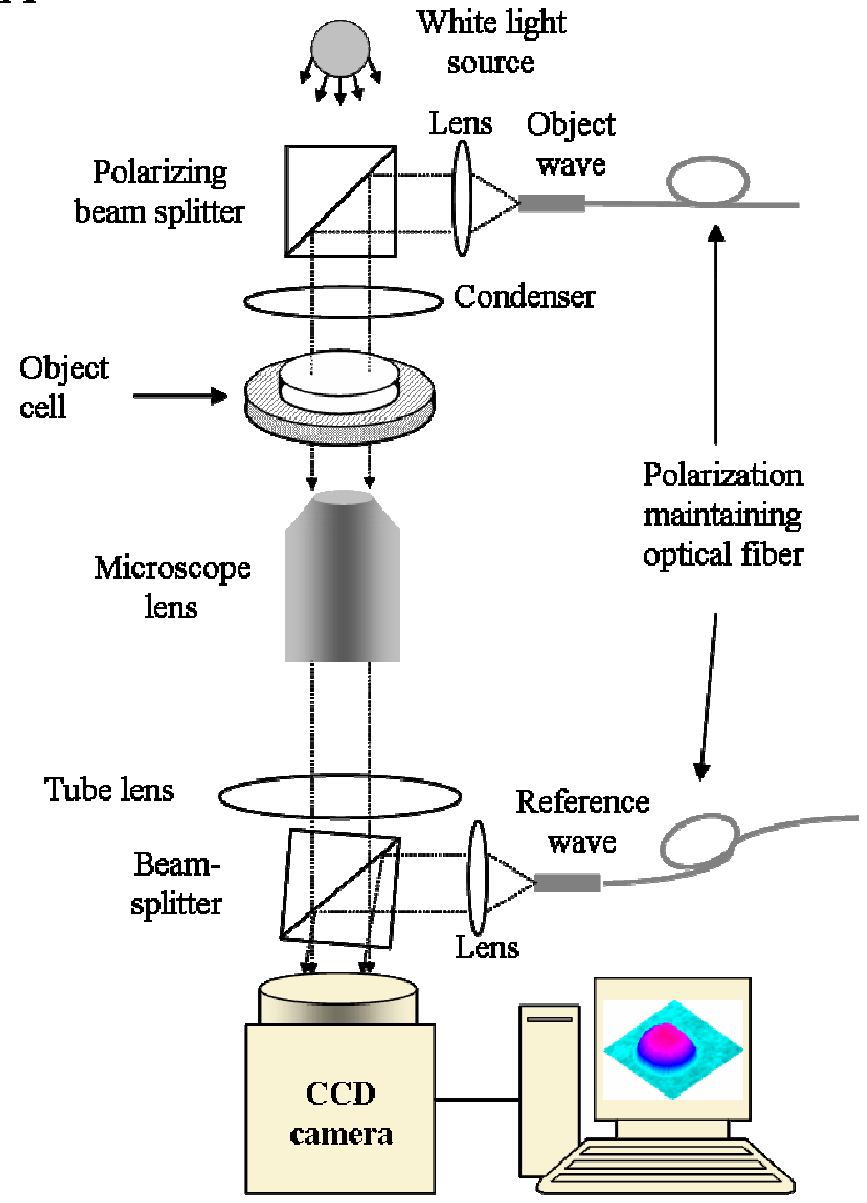

B

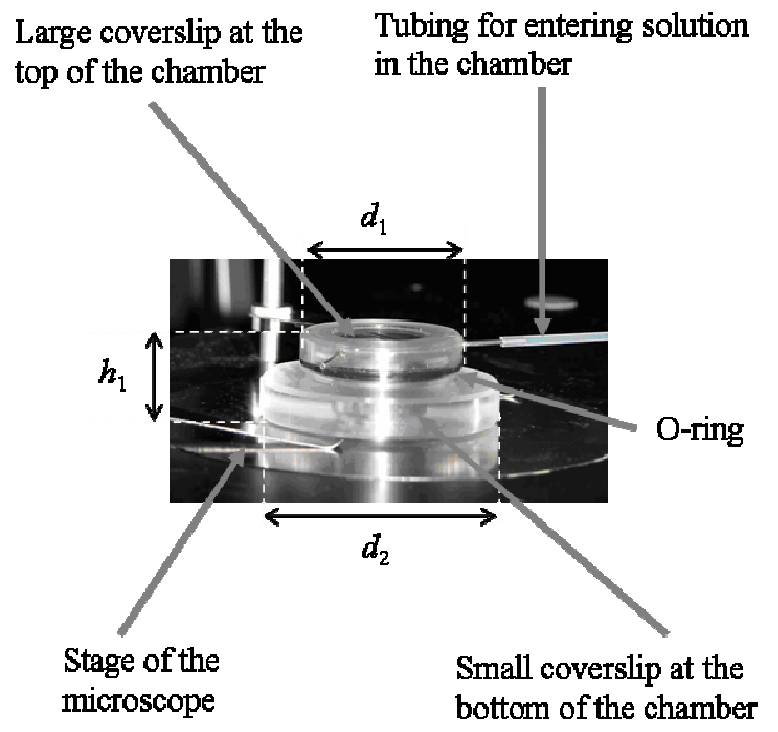

Figure 1: (A) Digital holographic microscopy setup in inverse transmission light arrangement. The object cell is depicted in B. (B) Measurement cell used during the shrinking process of the giant liposomes on the stage of the microscope. The set of dimension $\left(d_{1}\right.$, $\left.d_{2}, h_{1}\right)$ is $(35,52,8 \mathrm{~mm})$ with an internal volume of the chamber of $2.5 \mathrm{~mL}$.

\section{RESULTS AND DISCUSSION}

\subsection{Observation of liposomes}

Once the liposomes had been rehydrated in $0.1 \mathrm{M}$ sucrose, giant unilamellar liposomes with diameters of tens of micrometers were found. The observed liposome diameters ranged from 20 to $80 \mu \mathrm{m}$. Fig. 2A shows representative giant vesicles stained with fluorescein dye. Based on the quantitative fluorometric estimation of the number of bilayers from Akashi et $a l .{ }^{31}$, the liposomal membranes were judged to be unilamellar. The TEM image of two adjacent giant liposomes, depicted in Fig. 2B, confirmed this hypothesis. Indeed, the lipid bilayer membranes of two different liposomes were found to be correctly amplified on a scale bar of $0.2 \mu \mathrm{m}$. The concentration of sucrose inside the vesicles was later increased to $2 \mathrm{M}$ and gave the same results regarding the unilamellarity of the membrane.

A giant multilamellar liposome is depicted in Fig. 3. The image, obtained by TEM, reveals the assembly of multiple lipid bilayer membranes. There are two possible explanations for the liposome assembly shown in Fig. 3. The first possibility is that the sonication time of $15 \mathrm{~min}$ was not long enough to allow the multiwalled liposomes to become unilamellar. The second possibility is that the dehydration / rehydration step led to the spreading of several lipid layers and the formation 
of larger but multilamellar liposomes. Incorporation of charged biomimetic lipids in the starting solution could be helpful to force the membranes to repel each other by electrostatic forces. The diameter of the vesicle shown in Fig. 3 is approximately $2 \mu \mathrm{m}$. This size does not correspond to a giant liposome (i.e. $>20 \mu \mathrm{m}$ ) because the section where the liposomes has been sliced is most probably at the top or at the bottom of the liposome and not near the diameter.

\section{A}

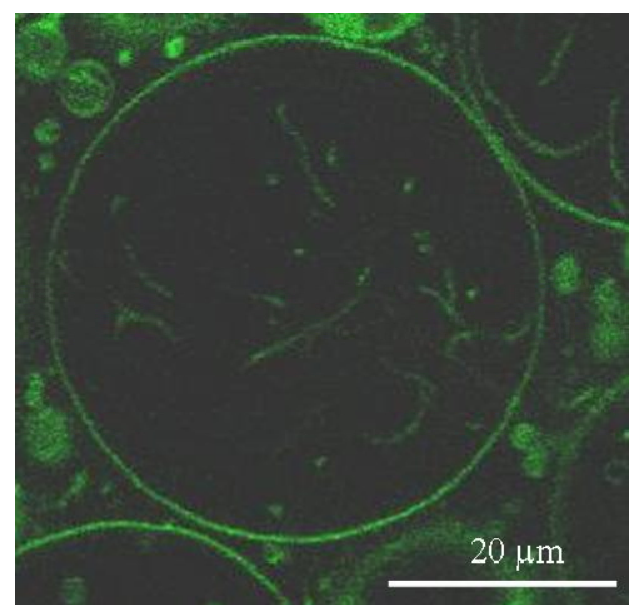

B

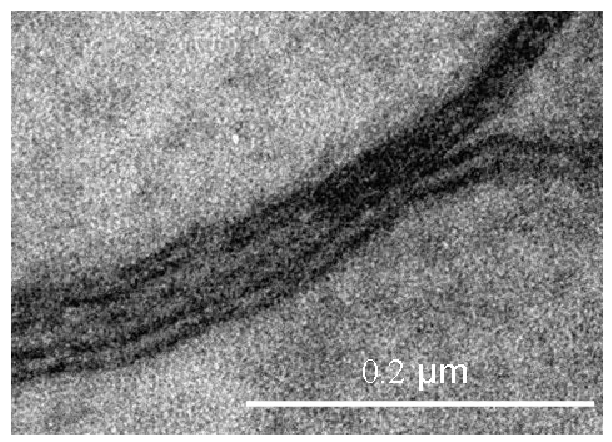

Figure 2: (A) Fluorescent image of giant unilamellar liposomes rehydrated in $0.1 \mathrm{M}$ sucrose (10\% cholesterol w/w) and stained with fluorescein dye. The picture was captured through a $60 \times$ objective on the laser scanning confocal microscope and the scale bar corresponds to $20 \mu \mathrm{m}$. (B) TEM image of a transversal section (130 nm thick) taken at the extremities of two adjacent giant liposomes, depicting their lipid bilayer membrane. The scale bar is $0.2 \mu \mathrm{m}$.

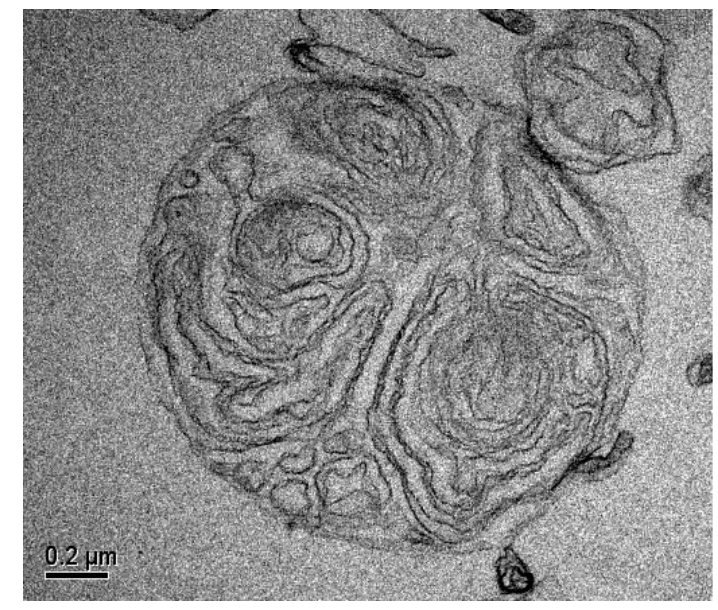

Figure 3: TEM image of a giant multilamellar liposome containing $10 \%$ cholesterol $\mathrm{w} / \mathrm{w}$. The scale bar is $0.2 \mu \mathrm{m}$.

\subsection{Digital holographic microscopy}

The digital holographic microscopy system has enabled high resolution quantitative phase contrast measurement of a giant unilamellar liposome $(\varnothing 20 \mu \mathrm{m})$.

However, to achieve these measurements with unilamellar liposomes it was necessary to modify the liquid medium inside the liposome in order to enhance the difference in refractive index with the external liquid. This is because the 
quantitative phase contrast generated increases with the difference in refractive index between the internal and external solutions. Although the refractive index of the lipid bilayer is $(1.42-1.45)^{33}$, the bilayer thickness is less that the current zresolution of the holographic microscope and is thus "invisible" under these conditions. Because the multilamellar liposomes are filled with lipid bilayers and the external liquid has a refractive index close to 1.333 , no difficulty was encountered in measuring the giant multilamellar liposomes, as seen in Fig. 4. This was not the case for the giant unilamellar liposomes, referred to as "invisible liposomes".

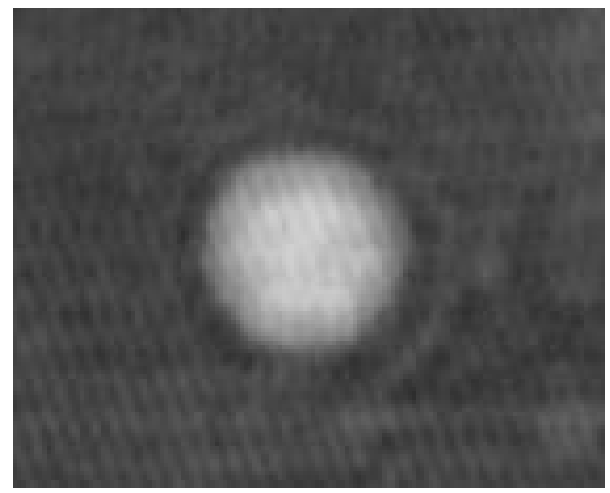

Figure 4: Reconstructed phase image of a giant multilamellar liposome (10 \% cholesterol w/w $)$

On the basis of Table 1, giant unilamellar liposomes prepared in $0.1 \mathrm{M}$ sucrose solution and diluted in an aqueous solution of $0.1 \mathrm{M}$ glucose gave a refractive index difference of 0.003 which is far below the necessary value of 0.1 for the holographic microscope. To achieve the required difference in refractive indices, an aqueous solution of $2 \mathrm{M}$ sucrose with a refractive index of 1.405 was used as the internal solution of the liposome. As the refractive index difference between sample and surrounding medium increased, the reconstructed quantitative phase image of the giant unilamellar liposomes became clearer with more distinct features.

\begin{tabular}{l|l|l}
\hline Chemicals & Concentration $\left(\mathbf{m o l} \cdot \mathbf{L}^{\mathbf{1}}\right)$ & Refractive index \\
\hline Sucrose & 0.1 & 1.339 \\
Glucose & 0.1 & 1.336 \\
Sucrose & 2 & 1.405 \\
$\mathrm{NaCl}$ & 1 & 1.344 \\
Pure water & - & 1.333 \\
lipid & - & $1.42-1.45$ \\
\hline
\end{tabular}

Table 1: Refractive index of solutes used as internal or external solution in liposome preparation measured with a refractometer at $20^{\circ} \mathrm{C}$. The refractive index of the lipid membrane is given as seen in Ardhammar et al. ${ }^{33}$.

Once we have been able to visualize the giant unilamellar liposomes with the holographic microscope, we next measured the structural changes in liposomes induced by osmotic stresses. To realize this experiment, a $\mathrm{NaCl}$ solution $(2 \mathrm{M})$ was added progressively to the surrounding medium of the liposome, developing a shrinkage of the vesicle. Fig. 5a shows the reconstructed quantitative phase images of a giant unilamellar liposome after different times (i.e. $t=0,83,116,140 \mathrm{~s}$ ), $t$ $=0$ being the time at which the stimulation with $\mathrm{NaCl}$ began. Fig. $5 \mathrm{~b}$ illustrates the reconstructed phase contrast images as pseudo three dimensional representations. At $t=83 \mathrm{~s}$, the liposome began to deform under the influence of increasing salt concentration. The shrinking process forced the internal solution to flow out developing a compression of the lipid membrane of the liposome as seen at $t=116 \mathrm{~s}$. At $t=140 \mathrm{~s}$, the lipid was found to be present only in small amount in the 
phase contrast image. No invagination of the membrane was observed at any time. This observation indicates that either the lipid membrane is totally compressed or that the lipid is dispersed in the surrounding medium.

\section{a - Reconstructed quantitative phase image}
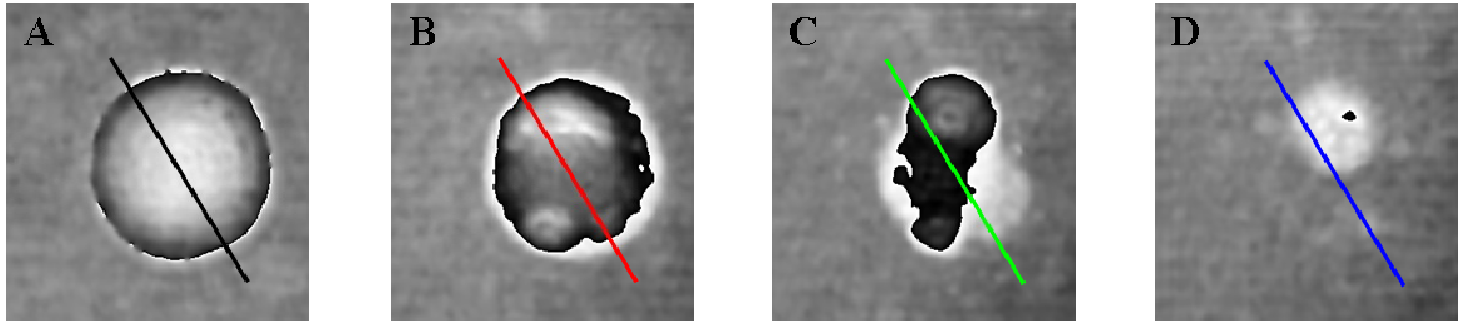

\section{b - Pseudo 3D representation}
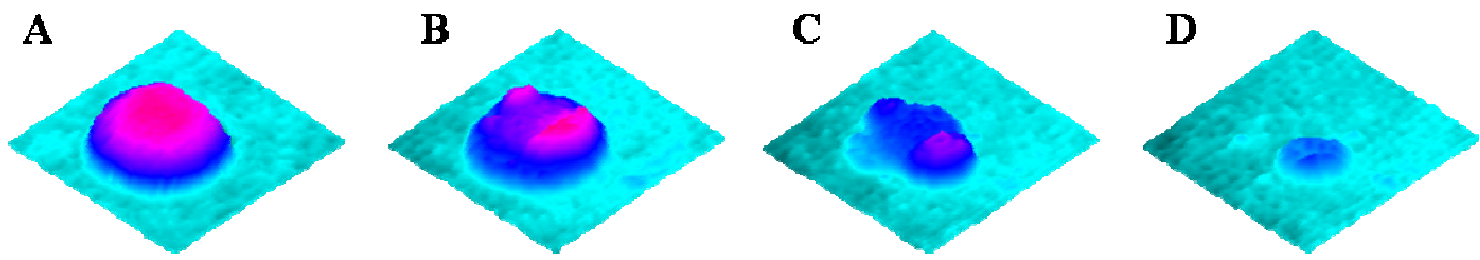

Figure 5: (a) Reconstructed quantitative phase images modulo $2 \pi$ and (b) pseudo 3D representations of the unwrapped phase images of a giant unilamellar liposome $(10 \%$ cholesterol w/w) during its shrinking in $2 \mathrm{M} \mathrm{NaCl}$ solution at different times: (A) $0 \mathrm{~s}$, (B) $83 \mathrm{~s}$, (C) $116 \mathrm{~s}$, (D) $140 \mathrm{~s}$. Lines through the reconstructed quantitative phase images of the liposome are cross sections needed to plot the graph depicted on Fig. 6.

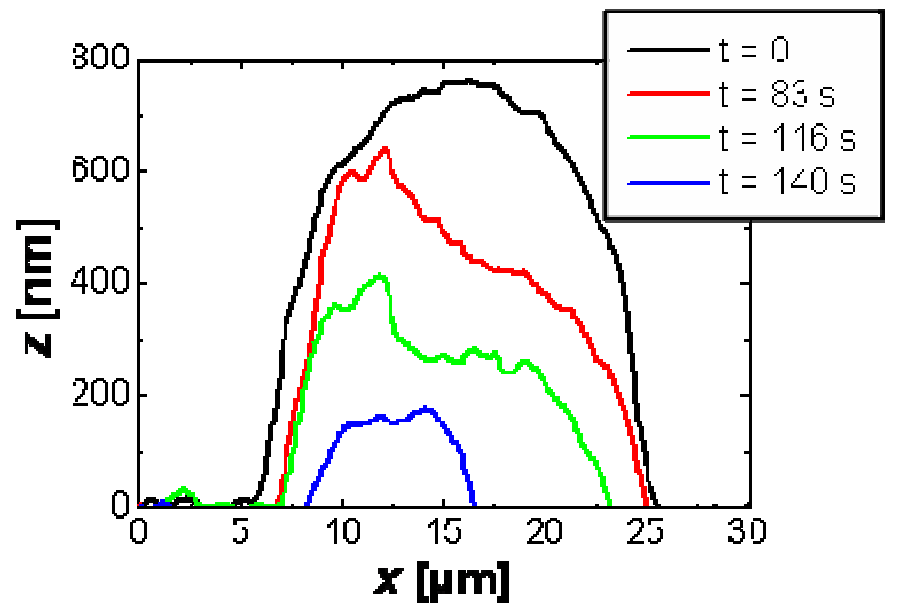

Figure 6. Quantitative analysis of the morphology change of the giant liposome via cross sections through the reconstructed phase images depicted in Fig. 5a. 
To quantify changes in optical path length affected by volume and refractive index changes, cross sections through the quantitative phase images were used (Fig. 5a) and a graph of the optical path length, $z$, versus the cross section, $x$, was plotted in Fig. 6, for the different times (i.e. $t=0,83,116,140 \mathrm{~s}$ ). The picture is a representation of the change in thickness of the liposome. A decrease in the optical path length as well as in the diameter of the liposome (through the cross section) was observed. However, the decrease was not symmetrical and seemed to be slower in the left part of the cross section (between 5 and $15 \mu \mathrm{m}$ ).

This distorted shape where the optical path length lent toward the left at a time equals to 83, 116 and $140 \mathrm{~s}$ was found to be suppressed when $40 \%$ cholesterol was included in the lipid bilayer membrane compared to $10 \%$ [data not shown]. It is well known that cholesterol, major component in most mammalian membranes, introduces conformational ordering of the lipid chains and increases the mechanical stiffness of the membrane while keeping the membrane fluid ${ }^{34}$.

\section{CONCLUSIONS}

In the present paper, the successful preparation of giant unilamellar liposomes has been shown as well as the observation of the lipid bilayer membrane. Digital holographic microscopy has been applied to investigate the structural properties of the giant liposomes. In contrast to standard phase contrast microscopy, quantitative phase imaging with high resolution can be obtained using this digital holographic system. This feature has allowed us to quantify the effect of salt on the deformation of a giant unilamellar liposome.

The presented results are preliminary and the application of digital holographic microscopy to unilamellar liposomes is novel. It would be of great interest to apply repetitive osmotic stresses such as a shrinking in $2 \mathrm{M} \mathrm{NaCl}$ followed by a swelling in pure water to see if the lipid bilayer membrane becomes again unilamellar. Similarly, it would be interesting to investigate if the deformation shapes of the liposome observed in Fig. 5 are due to the molecules of sucrose which are present in large excess in the liposome. For this analysis, the area difference elasticity model would be useful.

Acknowledgements: The authors wish to acknowledge $\mathrm{OzNano}_{2}$ life for financial support. $\mathrm{OzNano}$ life is a project (number CG060027) proudly supported by the International Science Linkages program under the Australian Government's Innovation statement Backing Australia's Ability.

\section{REFERENCES}

1. D. Carl, B. Kemper, G. Wernicke and G. von Bally, "Parameter-optimized digital holographic microscope for high-resolution living-cell analysis", Applied Optics, 43, 6536-6544 (2004)

2. W. D. Belt, "The origin of adrenal cortical mitochondria and liposomes: a preliminary report", Journal of Biophysical and Biochemical Cytology, 4, 337-340 (1958)

3. F. F. Schlunk and B. Lombardi, "Liver liposomes. I. Isolation and chemical characterization", Laboratory Investigation, 17, 30-38 (1967)

4. $\quad$ F. Schuber, A. Kichler, C. Boeckler and B. Frisch, "Liposomes: from membrane models to gene therapy", Pure and Applied Chemistry, 70, 89-96 (1998)

5. $\quad$ H. H. Sharata and K. H. Katz, "Liposomes", International Journal of Dermatology, 35, 761-769 (1996)

6. M. A. Jones, P. K. Kilpatrick and R. G. Carbonell, "Competitive immunosorbent assays using ligand-enzyme conjugates and bifunctional liposomes: Theory and experiment", Biotechnology Progress, 12, 519-526 (1996)

7. G. Poste, "The interaction of lipid vesicles (liposomes) with cultured cells and their use as carriers for drugs and macromolecules", Liposomes in Biological Systems, p 101-151, Wiley, New York, 1980

8. M. Ranson, A. Howell, S. Cheeseman and J. Margison, "Liposomal drug delivery", Cancer Treatment Reviews, 22, 365-379 (1996)

9. M. Putman, H. W. van Veen and W. N. Konings, "Molecular properties of bacterial multidrug transporters", Microbiology and Molecular Biology Reviews, 64, 672-+ (2000)

10. F. Szoka and D. Papahadjopoulos, "Comparative properties and methods of preparation of lipid vesicles (liposomes)", Annual Review of Biophysics and Bioengineering, 9, 467-508 (1980)

11. R. R. C. New, Liposomes, Oxford University Press, Oxford, 1990

Proc. of SPIE Vol. 6036 60361R-8 
12. N. Rodriguez, F. Pincet and S. Cribier, "Giant vesicles formed by gentle hydration and electroformation: A comparison by fluorescence microscopy", Colloids and Surfaces B-Biointerfaces, 42, 125-130 (2005)

13. F. M. Menger and M. I. Angelova, "Giant vesicles: Imitating the cytological processes of cell membranes", Accounts of Chemical Research, 31, 789-797 (1998)

14. F. M. Menger and J. S. Keiper, "Giant vesicles: Micromanipulation of membrane bilayers", Advanced Materials, 10, 888-+ (1998)

15. M. K. Doeven, J. H. A. Folgering, V. Krasnikov, E. R. Geertsma, G. van den Bogaart and B. Poolman, "Distribution, lateral mobility and function of membrane proteins incorporated into giant unilamellar vesicles", Biophysical Journal, 88, 1134-1142 (2005)

16. J. Korlach, P. Schwille, W. W. Webb and G. W. Feigenson, "Characterization of lipid bilayer phases by confocal microscopy and fluorescence correlation spectroscopy", Proceedings of the National Academy of Sciences of the United States of America, 96, 8461-8466 (1999)

17. L. A. Bagatolli and E. Gratton, "Two photon fluorescence microscopy of coexisting lipid domains in giant unilamellar vesicles of binary phospholipid mixtures", Biophysical Journal, 78, 290-305 (2000)

18. M. I. Angelova and I. Tsoneva, "Interactions of DNA with giant liposomes", Chemistry and Physics of Lipids, 101, 123-137 (1999)

19. Y. Yamashita, S. M. Masum, T. Tanaka and M. Yamazaki, "Shape changes of giant unilamellar vesicles of phosphatidylcholine induced by a de novo designed peptide interacting with their membrane interface", Langmuir, 18, 9638-9641 (2002)

20. S. Chiruvolu and J. A. N. Zasadzinski, "Membrane Elasticity Effects on Permeability Measurements in Vesicles", Aiche Journal, 39, 647-652 (1993)

21. P. Meleard, C. Gerbeaud, T. Pott, L. Fernandez-Puente, I. Bivas, M. D. Mitov, J. Dufourcq and P. Bothorel, "Bending elasticities of model membranes: Influences of temperature and sterol content", Biophysical Journal, 72, 2616-2629 (1997)

22. A. Viallat, J. Dalous and M. Abkarian, "Giant lipid vesicles filled with a gel: Shape instability induced by osmotic shrinkage", Biophysical Journal, 86, 2179-2187 (2004)

23. B. L. S. Mui, H. G. Dobereiner, T. D. Madden and P. R. Cullis, "Influence of Transbilayer Area Asymmetry on the Morphology of Large Unilamellar Vesicles", Biophysical Journal, 69, 930-941 (1995)

24. L. A. Bagatolli and E. Gratton, "Two-photon fluorescence microscopy observation of shape changes at the phase transition in phospholipid giant unilamellar vesicles", Biophysical Journal, 77, 2090-2101 (1999)

25. K. Akashi, H. Miyata, H. Itoh and K. Kinosita, "Formation of giant liposomes promoted by divalent cations: Critical role of electrostatic repulsion", Biophysical Journal, 74, 2973-2982 (1998)

26. P. Meleard, J. F. Faucon, M. D. Mitov and P. Bothorel, "Pulsed-Light Microscopy Applied to the Measurement of the Bending Elasticity of Giant Liposomes", Europhysics Letters, 19, 267-271 (1992)

27. G. von Bally, Holography in Medicine and Biology, Springer-Verlab, Berlin, 1979

28. B. Kemper, D. Carl, S. Knoche, G. Thien and G. von Bally, "Holographic interferometric microscopy systems for the application on biological samples", Proceedings of the International Conference Photonics Europe, 26-30 April 2004, SPIE, 5457, 581-588 (2004)

29. H. Nagase, H. Ueda and M. Nakagaki, "Effect of water on lamellar structure of DPPC/sugar systems", Biochimica et Biophysica Acta-Biomembranes, 1328, 197-206 (1997)

30. M. A. Kiselev, S. Wartewig, M. Janich, P. Lesieur, A. M. Kiselev, M. Ollivon and R. Neubert, "Does sucrose influence the properties of DMPC vesicles?" Chemistry and Physics of Lipids, 123, 31-44 (2003)

31. K. Akashi, H. Miyata, H. Itoh and K. Kinosita, "Preparation of giant liposomes in physiological conditions and their characterization under an optical microscope", Biophysical Journal, 71, 3242-3250 (1996)

32. Y. Yamashita, M. Oka, T. Tanaka and M. Yamazaki, "A new method for the preparation of giant liposomes in high salt concentrations and growth of protein microcrystals in them", Biochimica et Biophysica ActaBiomembranes, 1561, 129-134 (2002)

33. M. Ardhammar, P. Lincoln and B. Norden, "Invisible liposomes: Refractive index matching with sucrose enables flow dichroism assessment of peptide orientation in lipid vesicle membrane", Proceedings of the National Academy of Sciences of the United States of America, 99, 15313-15317 (2002)

34. S. Raffy and J. Teissie, "Control of lipid membrane stability by cholesterol content", Biophysical Journal, 76, 2072-2080 (1999) 